\title{
GMR
}

\section{Cloning and characterization of SPL-family genes in the peanut (Arachis hypogaea L.)}

\author{
M. Li ${ }^{1 *}$, S.Z. Zhao ${ }^{1 *}$, C.Z. Zhao', Y. Zhang ${ }^{1}$, H. Xia' ${ }^{1}$, J. Lopez-Baltazar', S.B. Wan ${ }^{1}$ \\ and X.J. Wang ${ }^{1}$ \\ ${ }^{1}$ Biotechnology Research Center, Shandong Academy of Agricultural Sciences, \\ Shandong Provincial Key Laboratory of Crop Genetic Improvement, \\ Ecology and Physiology, Jinan, Shandong Province, China \\ ${ }^{2}$ Instituto Tecnológico del Valle de Oaxaca, Oaxaca, Mexico \\ *These authors contributed equally to this study. \\ Corresponding authors: X.J. Wang / S.B. Wan \\ E-mail: xingjunw@hotmail.com / wanshb@saas.ac.cn \\ Genet. Mol. Res. 15 (1): gmr.15017344 \\ Received September 28, 2015 \\ Accepted November 13, 2015 \\ Published February 19, 2016 \\ DOI http://dx.doi.org/10.4238/gmr.15017344
}

ABSTRACT. SQUAMOSA promoter-binding protein-like (SPL) proteins play crucial roles in plant growth, development, and responses to environmental stressors. The peanut (Arachis hypogaea L.) is a globally important oil crop. In this study, we cloned the full-length CDNA of 15 SPLs in the peanut by transcriptome sequencing and rapid amplification of cDNA ends, and analyzed their genomic DNA sequences. cDNA lengths varied significantly, from 369 to $3102 \mathrm{bp}$. The SBP domain of the peanut SPL proteins was highly conserved compared to SPLs in other plant species. Based on their sequence similarity to SPLs from other plant species, the peanut $S P L s$ could be grouped into five subgroups. In each subgroup, lengths of individual genes, conserved motif numbers, and distribution patterns were similar. Seven of the SPLs were predicted to be targets of miR156. The SPLS were ubiquitously expressed in the roots, leaves, flowers, gynophores, and seeds, with different expression levels and accumulation patterns. Significant differences in the expression of most of the $S P L s$ were observed between juvenile and adult leaves, suggesting that 
they are involved in developmental regulation. Dynamic changes occurred in transcript levels at stage 1 (aerial grown green gynophores), stage 2 (gynophores buried in soil for about three days), and stage 3 (gynophores buried in soil for about nine days with enlarged pods). Possible roles that these genes play in peanut pod initiation are discussed.

Key words: SQUAMOSA promoter-binding protein-like protein; Peanut; Gene expression; Pod development

\section{INTRODUCTION}

SQUAMOSA (SQUA) promoter-binding protein-like (SPL) proteins are plant-specific transcription factors that play important roles in plant growth and development. Two SPL genes, SBP1 and SBP2, which are highly homologous to AmSBP1, were first isolated from Antirrhinum majus by their capacity to interact with a promoter sequence element of SQUA (Klein et al., 1996). Genome-wide analysis has revealed that 17 members of the SPL family genes are present in Arabidopsis (Birkenbihl et al., 2005; Guo et al., 2008). To date, a large number of SPLs have been discovered in various plant species, such as green algae, moss, tomato, rice, maize, and silver birch (Moreno et al., 1997; Lännenpää et al., 2004; Kropat et al., 2005; Xie et al., 2006; Riese et al., 2007; Salinas et al., 2012).

The SBP-box proteins encoded by SPLs all contain a highly conserved DNA-binding SBP domain, which includes approximately 76 amino acid residues and two zinc-binding motifs (Birkenbihl et al., 2005; Kropat et al., 2005; Xie et al., 2006; Riese et al., 2007; Salinas et al., 2012). A bipartite nuclear localization signal (NLS) sequence is located at the C-terminus of the SBP domain, which overlaps with the DNA-binding domain and the second zinc-binding motif (Birkenbihl et al., 2005). Plant SPL proteins can be divided into different subgroups, based on the SQUA promoter-binding domain sequence, e.g., there are six subgroups in rice, seven in the grapevine, and eight in the tomato (Xie et al., 2006; Salinas et al., 2012; Hou et al., 2013). The gene structures of these SPLs are different in terms of intron and exon number, as well as coding region length.

An increasing number of microRNAs (miRNAs) have been identified and characterized, and shown to have crucial gene regulation functions in plants. miRNAs are small, non-coding RNA molecules (20-24 nucleotides) that can bind to their target mRNAs by imperfect base pairing for transcript cleavage, and decrease protein levels by translational repression (Voinnet, 2009). Approximately half of the target genes of miRNAs are transcription factors. As a gene family that encodes transcription factors, more than half of SBP-box genes have been found to be targeted by miR156 and miR157 in some species. For example, the majority (11/17) of Arabidopsis SPLs contain miR156/157-binding sites that are located in the coding region or the 3' untranslated region (UTR), indicating that miRNAs could regulate SPLs by mRNA cleavage and translation inhibition (Rhoades et al., 2002; Gandikota et al., 2007; Xing et al., 2010). In rice, 11 of 19 SPLs contain sequences that are complementary to mature miR156. The target sites of nine SPLs are located in the coding region downstream of the SBP domain. The target sites of two SPLs are located in the 3' UTR (Xie et al., 2006), and 15 of 27 SBP-box gene family members in apples contain putative miR156 targeting sites, all of which are located in the coding region (Li et al., 2013). Therefore, miR156 could downregulate SPL expression by mRNA degradation or translation inhibition (Wu and Poethig, 2006).

Previous studies have demonstrated the involvement of SPLs in the regulation of many 
aspects of plant development, such as leaf shape and size, trichome formation, tiller number, apical dominance, inflorescence architecture, pollen development, fertility, and fruit ripening (Unte et al., 2003; Schwarz et al., 2008; Shikata et al., 2009; Usami et al., 2009; Wu et al., 2009; Xing et al., 2010). SPLs are also involved in the regulation of the gibberellin (GA) response (Zhang et al., 2007) and copper homeostasis (Yamasaki et al., 2009). A few studies have found that SBPbox genes are responsible for embryo development. For example, more than half of OsSPLs are highly expressed in young panicles (Xie et al., 2006). Furthermore, OsSPL14 (Miura et al., 2010) and OsSPL16 (Wang et al., 2012) play crucial roles in determining grain quality and yield in rice. tsh4 (tassel sheath4), a member of the maize SBP-box gene family, regulates bract development, and is required for branch meristem initiation and maintenance (Chuck et al., 2010). In addition, a series of studies have shown that SPLs play important roles in regulating plant developmental phase transition. For example, AtSPL9 and AtSPL10 promote the transition from the juvenile to the adult phase by upregulating the expression of miR172, and thereby repressing its targets, TARGET OF EAT 1 (TOE1) and TOE2, in Arabidopsis (Wu et al., 2009). Most miR156-targeted SPLs accelerate flowering by directly activating flower-promoting MADS box genes, such as FRUITFULL (FUL), SUPPRESSOR OF OVEREXPRESSION OF CONSTANS 1 (SOC1), AGAMOUS-LIKE (AGL42), and APETALA 1 (AP1). In addition, SPL3 directly activates $\angle E A F Y$ (LFY), which is a master regulator of meristem identity transition, in order to control the time of flowering (Weigel et al., 1992; Yamaguchi et al., 2009). In perennial trees, vegetative to adult phase transition is controlled by SPLs that are regulated by miR156. The overexpression of miR156 results in the reduced expression of miR156targeted SPLs, and significantly prolongs the juvenile phase (Wang et al., 2011).

Although the roles of plant-specific SBP-box genes in development have been substantially elucidated in Arabidopsis and other plants, there is little information available concerning the function of SBP-box genes in the peanut (Arachis hypogaea L.). Peanut is an important global oil crop and protein source. Peanut plant flowers and finishes pollination above ground as do other plants, while the ovule-carrying peg (gynophore) starts to form above ground and then grows downwards to bury the fertilized ovule in the soil for pod development (Thompson et al., 1985; Xia et al., 2013; Zhu et al., 2014). In this study, we identified 15 potential peanut SPLs that all contained a conserved SBP domain. According to the deduced amino acid, they were named AhSPL1, AhSPL2-1, AhSPL2-2, AhSPL3-1, AhSPL3-2, AhSPL4, AhSPL6, AhSPL7, AhSPL8, AhSPL9, AhSPL12, AhSPL13, AhSPL14, AhSPL16, and AhSPL18. The gene structures and expression patterns of these SPLs in peanut tissues at different developmental stages were analyzed, and possible roles of SPLs in peanut growth and development are discussed.

\section{MATERIAL AND METHODS}

\section{Plant materials}

Roots, juvenile stems, and juvenile leaves were collected from two-week-old seedlings of the peanut cultivar, 'Luhua-14', and adult stems, leaves, and flowers were harvested at the full-bloom stage. Gynophores were collected at 0, 3, and 9 days after burial in the soil, and the seeds were harvested at maturity. Fresh samples were frozen in liquid nitrogen and stored at $-80^{\circ} \mathrm{C}$ for RNA preparation.

Downward-growing green gynophores, which were $3-5 \mathrm{~cm}$ in length, were categorized as stage 1 (S1). Stage 2 gynophores (S2) were buried in soil for about three days, and had thicker diameters than S1 gynophores. S2 gynophores were white, and enlargement of the ovary region 
was not observed. Stage 3 gynophores (S3) were buried in soil for about nine days, and their ovary regions were enlarged but still small.

\section{RNA isolation and first-strand cDNA synthesis}

Total RNA was extracted from different tissues using a modified CTAB method, with isopropanol instead of lithium chloride for the RNA precipitation. RNase-free DNase I (TaKaRa) was added to remove contaminating genomic DNA. About $1 \mu \mathrm{g}$ of total RNA was used for the first-strand cDNA synthesis, following the protocol of the Revert Aid First Strand cDNA Synthesis Kit (Fermentas).

\section{Gene cloning and sequencing}

The forward degenerate primer (5'-GRTTYTGYCARCARTGYAGCAG-3') was designed based on the conserved SBP-box domain, and the reverse primer (5'-TGACAGAAGAGAGAGAG CAC-3') was designed based on the conserved miRNA156/157 target site. Subsequently, reverse transcription-polymerase chain reaction (RT-PCR) was conducted to clone the middle fragments of the SPLs. To obtain full-length cDNA sequences, 5'-rapid amplification of cDNA ends (5'-RACE) and $3^{\prime}-$ RACE were performed according to the manual of the SMARTer ${ }^{T M}$ RACE cDNAAmplification Kit (Clontech). Gene-specific primers (GSP), which were designed based on the middle fragments of the cloned SPLs, and a Universal Primer A Mix provided by the SMARTer ${ }^{T M}$ RACE CDNA Amplification Kit, were used for RACE-PCR. It was necessary to set up a nested PCR with an inner GSP and a Nested Universal Primer A.

Based on the peanut transcriptome sequencing, PCR primer pairs were designed to amplify the open reading frames (ORFs) of the SPLs. The PCR products were purified using a PCR Purification kit (Sangon Biotech, China), ligated into a pMD18-T simple vector (TaKaRa), and transformed into Escherichia coli $\mathrm{DH} 5 \alpha$ cells. Positive clones were selected and sequenced by the Sequencing Center, Shandong Academy of Agricultural Sciences, China.

\section{Multiple sequence alignments and phylogenetic and gene structure analyses}

To examine in detail the domain organization of SPL proteins in peanut, multiple sequence alignments of SBP domain sequences were performed using Clustal W (Thompson et al., 1994). Phylogenetic and molecular evolution analysis of the SPL proteins was conducted using MEGA 4.0 (Tamura et al., 2007). In an effort to gain an insight into the evolutionary relationships between SBPbox genes in various plant species, we selected 107 SBP-box genes from six species, including 75 from dicotyledonous plants (Arabidopsis, Vitis vinifera, Populus trichocarpa, and A. hypogaea), 19 from a monocotyledonous plant (Oryza sativa), and 13 from the moss Physcomitrella patens to include a non-flowering plant, and constructed a phylogenetic tree based on the highly conserved protein sequences of the SBP domain (about 76 amino acids), using the neighbor-joining method with 1000 bootstrap replications (Table S1). The gene structure display server program was used to illustrate exon-intron organization for individual SPLs by comparing the cDNA sequences with their corresponding genomic DNA sequences.

\section{Identification of conserved motifs}

Conserved motif analysis within the SBP-box family was performed using the MEME 
program (http://meme-suite.org/) with default settings, except an optimum motif width of between 6 and 200 residues and a maximum of 20 motifs.

\section{Gene expression analysis by quantitative real-time PCR}

Total RNA was extracted from different organs by the CTAB method. One microgram of total RNA was treated with DNase I and was then used for first-strand CDNA synthesis with an oligo(dT) primer using a TaKaRa PrimeScript ${ }^{\text {Tw }} 1$ st Strand cDNA Synthesis Kit. Subsequently, a quantitative real-time PCR was performed with a SYBR ${ }^{\circledR}$ Green PCR Master Mix (Roche) and an iCycler Thermal Cycler (Bio-Rad, Hercules, CA, USA). The oligonucleotide primers are listed in Table S2.

\section{RESULTS}

\section{Cloning the full-length cDNAs of peanut SPLs}

The full-length CDNAs of $15 \mathrm{SPL}$ family genes in peanut were cloned. Based on the sequence similarity to Arabidopsis SPLs, they were named AhSPL1, AhSPL2-1, AhSPL2-2, AhSPL3-1, AhSPL3-2, AhSPL4, AhSPL6, AhSPL7, AhSPL8, AhSPL9, AhSP12, AhSPL13, AhSP14, AhSPL16, and $A h S P L 18$. The cDNA lengths of the SBP proteins varied from 369 to $3102 \mathrm{bp}$. Their corresponding molecular weights ranged from 14 to $113 \mathrm{kDa}$. AhSPL1 was the longest SPL coding gene, with a CDNA length of $3102 \mathrm{bp}$ that encoded a protein of $113 \mathrm{kDa}$. AhSPL3-2 was the shortest SPL coding gene, with a cDNA length of only $369 \mathrm{bp}$ that encoded a protein of $14.3 \mathrm{kDa}$. The SPL family of proteins also exhibited a large range of isoelectric point values, which ranged from 5.83 to 9.78 .

Previous studies have demonstrated that some plant SPL genes are targets of miRNAs. By comparing the miR156 mature sequence to the $S P L$ sequences, we identified seven $S P L S$ (AhSPL2-1, AhSPL2-2, AhSPL6, AhSPL9, AhSPL13, AhSPL16, and AhSPL18) that were possible targets of miR156 (Figure 1). Interestingly, all of these AhSPLs, which were predicted to be miRNA targets, were clustered in G2 and G3 (see below). miR156 target site of AhSPL2-1 was located in the 3' UTR, while miR156 target sites of all other AhSPL2-1 were located in the coding regions. In Arabidopsis, SPLs are targeted by miR172; in the peanut, we did not find any SPL that was a target of the miR172 family.

\begin{tabular}{|c|c|c|}
\hline AnsPL2-1 & GATTTCCAGPGTGCTCTCTC & TCTTCTGTCAACCGAGTC \\
\hline AnSPL $2-2$ & GATGTCAGCDGTGCTCTCTC & TCTTCTGTCAACCAATTC \\
\hline AnSPL 6 & TTATCTAGCAGTGCTCTCT & TCTTCTGTAAGCTCAGTC \\
\hline AhspL9 & GACTCAAGCIGTGCTCTCT & TCTTCTGTCAAGCCAAAC \\
\hline AnSPL13 & GACTCTICCTGTGCTCTCT & TCTTCTGTCATCACCACA \\
\hline AnSPL16 & GATGCAGGTIGTGCTCTC & TCTTCTGTCAACAATACA \\
\hline AhSPL18 & GACTCTGGTTGTGCTCTCTC & TСTTCTGTCATCTCAATC \\
\hline AhmiR156a & .......gTGCTCtcto & тсттстято........ \\
\hline Ahmi156b-5p & GTGCTCTCI. & тсттстято. ........ \\
\hline AhmiR156c & GTGCTCTC: & TстTCTGTo........ \\
\hline Consensus & $\operatorname{gtgctctc}$ & tcttctgtc \\
\hline
\end{tabular}

Figure 1. Alignment of miR156 complementary sequences within peanut SPL genes. Complementary sequences are within coding regions, with the exception of AhSPL2-1 where it is located within the 3' UTR. Reverse complement sequences of the mature AhmiR156 genes are shown below the alignment for comparison. 


\section{Phylogenetic analysis of the SBP-domain family genes}

The unrooted phylogenetic tree indicated that the plant SBP-domain family of proteins is evolutionarily diverse (Figure 2). The 107 SBP sequences could be classified into the following six groups: AhSPL4, AhSPL3-1, AhSPL3-2, and AhSPL8 in G1; AhSPL2-1, AhSPL2-2, and AhSPL9 in G2; AhSPL18, AhSPL6, AhSPL16, and AhSPL13 in G3; AhSPL1, AhSPL12, and AhSPL14 in G4; and AhSPL7 in G6 (Figure 2). Based on the phylogenetic tree, most of the AhSPLs were more tightly grouped with grapevine, Arabidopsis, or poplar SBP-box proteins than with rice or moss SBP-box proteins. No peanut SBP protein was grouped with moss SBP proteins; therefore, peanut SBP proteins were grouped into five groups (Figure 2).

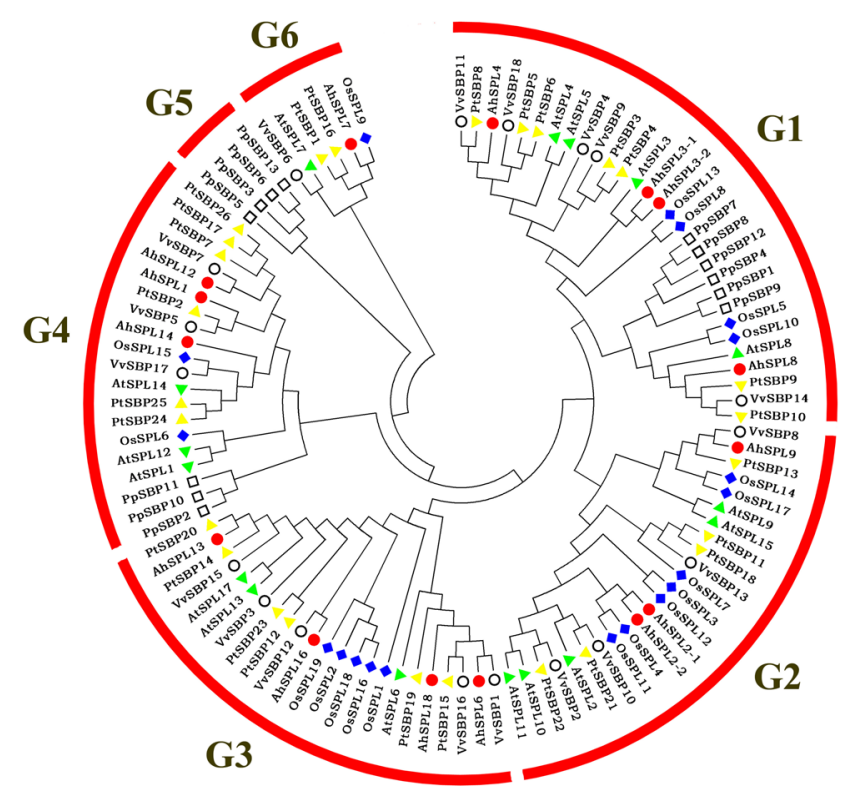

Figure 2. Phylogenetic analysis of peanut and other plant SBPs. Phylogenetic tree was constructed with SBP domain protein sequences from peanut (AhSPL), grape (VvSBP), moss (PpSBP), rice (OsSPL), popular (PtSBP) and Arabidopsis (AtSPL). Detail information of SBP sequences used to construct the phylogenetic tree was listed in $\underline{\text { Table S1. }}$.

To directly compare the 15 peanut SBP genes, a phylogenetic tree was constructed based on the full-length cDNA of the proteins (Figure 3). In general, the topology of the resulting phylogenetic tree was similar to that constructed with the 107 SBP-domain sequences from the six plant species (Figure 2). Interestingly, AhSPL8 and AhSPL9 were in G1 and G2, respectively, in the phylogenetic tree generated using the SBP-domain sequences from the six plant species (Figure 2); however, these two proteins were in G4 and G1, respectively, in the phylogenetic tree generated by full-length cDNA of peanut SPL genes (Figure 3).

The genomic sequences of the peanut SBP genes were obtained from the scaffolds generated by our peanut whole-genome sequencing project (data not shown). Exon-intron structures of all $15 \mathrm{AhSPLs}$ were generated based on their corresponding genome and coding sequences (Figure 3). The majority of the peanut SBP genes in the same subgroup bore a similar number of exons and introns, as well as ORF lengths. For example, genes within G4 contained 10 
exons, and encoded proteins with about 1000 amino acid residues. Genes in G1 contained two exons, and encoded proteins with fewer than 180 amino acid residues. However, AhSPL8 in G4 and AhSPL9 in G1 shared a similar exon-intron distribution to genes in G3 (Figure 3).

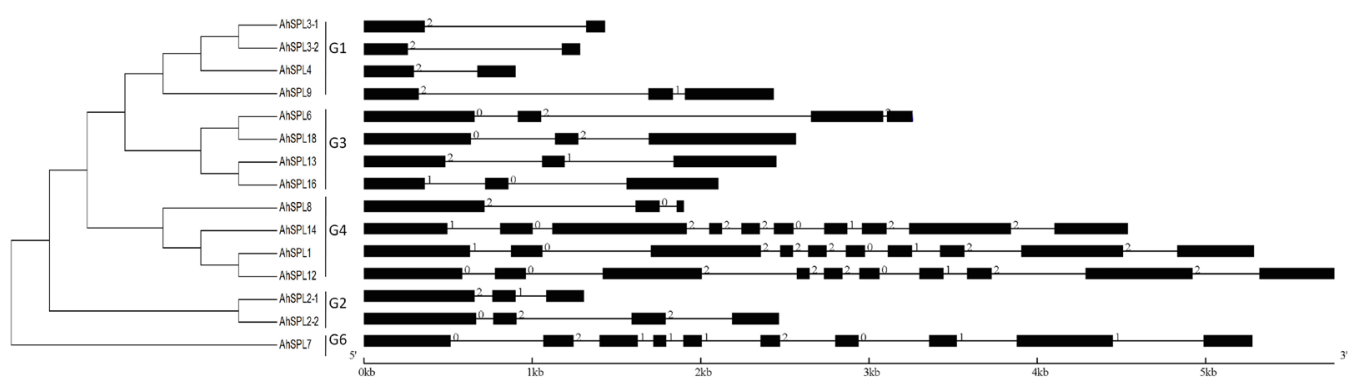

Figure 3. Phylogenetic relationships and gene structure of AhSPL genes. Phylogenetic analysis based on the fulllength cDNA of peanut SPLs. (B) Exon - intron structures of SPL genes from Arachis hypogaea. Exons and introns were represented by black boxes and black lines, respectively. The sizes of exons and introns can be estimated using the scale at the bottom. Numbers 0,1 , and 2 represent introns in phases 0,1 , and 2, respectively.

\section{Conserved sequences in the SBP domain}

All members of the SBP-box family contain a conserved SBP domain. We aligned the sequences of the SBP domain using Clustal X 1.83, and found that each SBP-domain sequence contained approximately 76 amino acid residues (Figure 4). The SBP-box proteins are plantspecific transcription factors. The structural basis for the sequence-specific DNA binding are two Zn-finger-like structures formed by the coordination of two zinc ions by conserved cysteine and histidine residues (Birkenbihl et al., 2005). The first Zn-finger-like structure (Zn-1 in Figure 4$)$ is $\mathrm{Cys}_{3}$ His-type, and the second ( $\mathrm{Zn}-2$ in Figure 4$)$ is Cys ${ }_{2}$ HisCys-type. In addition to the sequencespecific DNA-binding domain, the SBP domain has a highly conserved bipartite NLS (Birkenbihl et al., 2005), a key feature of transcription factors, and is located at the C-terminus of the SBP domain. Interestingly, the "His" in the first Zn-finger structure is replaced by a "Cys" in AhSPL7, and the "His" in the second Zn-finger structure is replaced by a Q residue in AhSPL6. A previous study demonstrated that the exchange of the "His" in the first Zn-finger with "A" leads to the complete loss of DNA-binding activity of Arabidopsis SPL1. The "His" replacement in the second Zn-finger also significantly affects the binding activity of SPL proteins (Birkenbihl et al., 2005). Therefore, there is little possibility that $A h S P L 7$ and $A h S P L 6$ act as functional transcription factors in peanut. The first and last $C$ residues in the second $Z n$-finger structure, which are critical for DNA binding, were completely conserved among the 15 peanut SBP domains. The NLS segments of the 15 peanut SBP domains were also highly conserved; however, the sequences flanking the SBP domain exhibited high sequence diversity (Figure 3). In addition to the two conserved Zn-finger structures and NLS segments, we also found other conserved sequences, e.g., the sequence between the second Zn-finger structure and the NLS.

\section{Conserved motifs in AhSPL proteins}

Twenty motifs were identified in the 15 SPL proteins (Table S3 $)$. A schematic representation of the conserved motifs among the SBP-box proteins was constructed (Figure 5), and the conserved 
amino acid sequences and motif lengths are shown in Table S3. The number of motifs in each SPL protein varied between 1 and 13, due to the different protein lengths. However, the number of motifs and the distribution order of different motifs in each SPL subgroup were similar (Figure 5). Motif 1, which was located in the SBP domain, was detected in all of the proteins. Some motifs were widely distributed in the SBP-box proteins, e.g., motifs $4,6,7,12$, and 18 , whereas others were only detected in specific subgroups, e.g., motif 15 was only in G4. This difference in motif number and distribution pattern might be associated with the function of each subgroup.

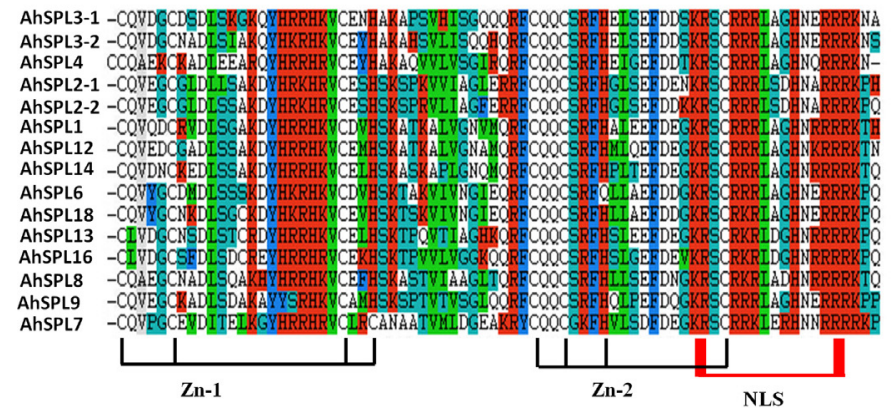

Figure 4. SBP domain sequence alignment in peanut. Multiple sequence alignment was performed using the Clustal X1.83 software. The two conserved Zn-finger structures and NLS were indicated.

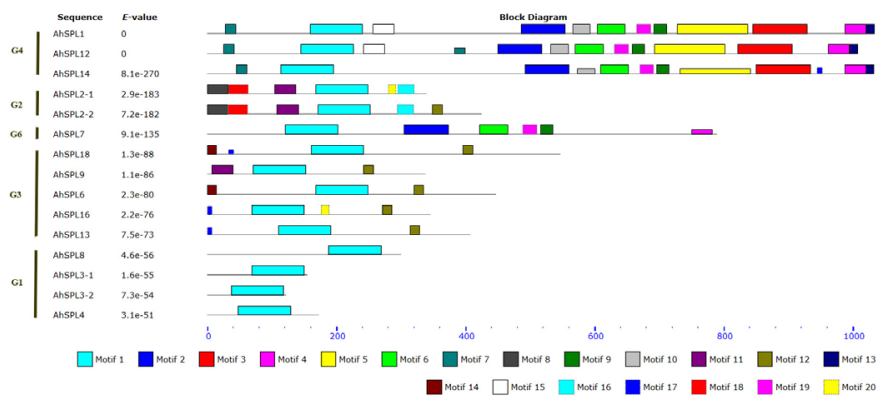

Figure 5. Distribution of 20 predicted conserved motifs in peanut SBP proteins. Motifs of Arachis hypogaea SBP proteins were identified by MEME program. The conserved amino acid sequences and length of each motif are shown in Table S3.

\section{Expression analysis of peanut SPLS}

The AhSPL coding genes were ubiquitously expressed in all of the tissues tested. However, the relative expression levels and patterns of preferential accumulation of the genes varied significantly. For example, AhSPL1 was detected in juvenile roots, stems, and leaves, and adult stems, leaves, flowers, S1, S2, S3 gynophores, and seeds, with relatively high expression levels in juvenile stems and leaves and adult leaves, S2 and S3 gynophores (Figure 6). The highest expression level detected in adult leaves was about four times higher than that in roots and S1 gynophores, in which the lowest expression levels were detected. However, AhSPL2-1 was preferentially expressed in stems and leaves (both juvenile and adult). The highest expression level was detected in adult leaves, which was more than 40 -fold higher than that in the roots. 
Expression levels in flowers, gynophores, and seeds were relatively low compared to those in leaves and stems (Figure 6). AhSPL2-2 exhibited similar expression patterns to AhSPL2-1; however, the AhSPL2-2 expression level in adult leaves was about 100-fold higher than that in the roots, juvenile leaves, and seeds. AhSPL2-2 accumulated at high levels in adult leaves, flowers, and S3 gynophores (Figure 6).

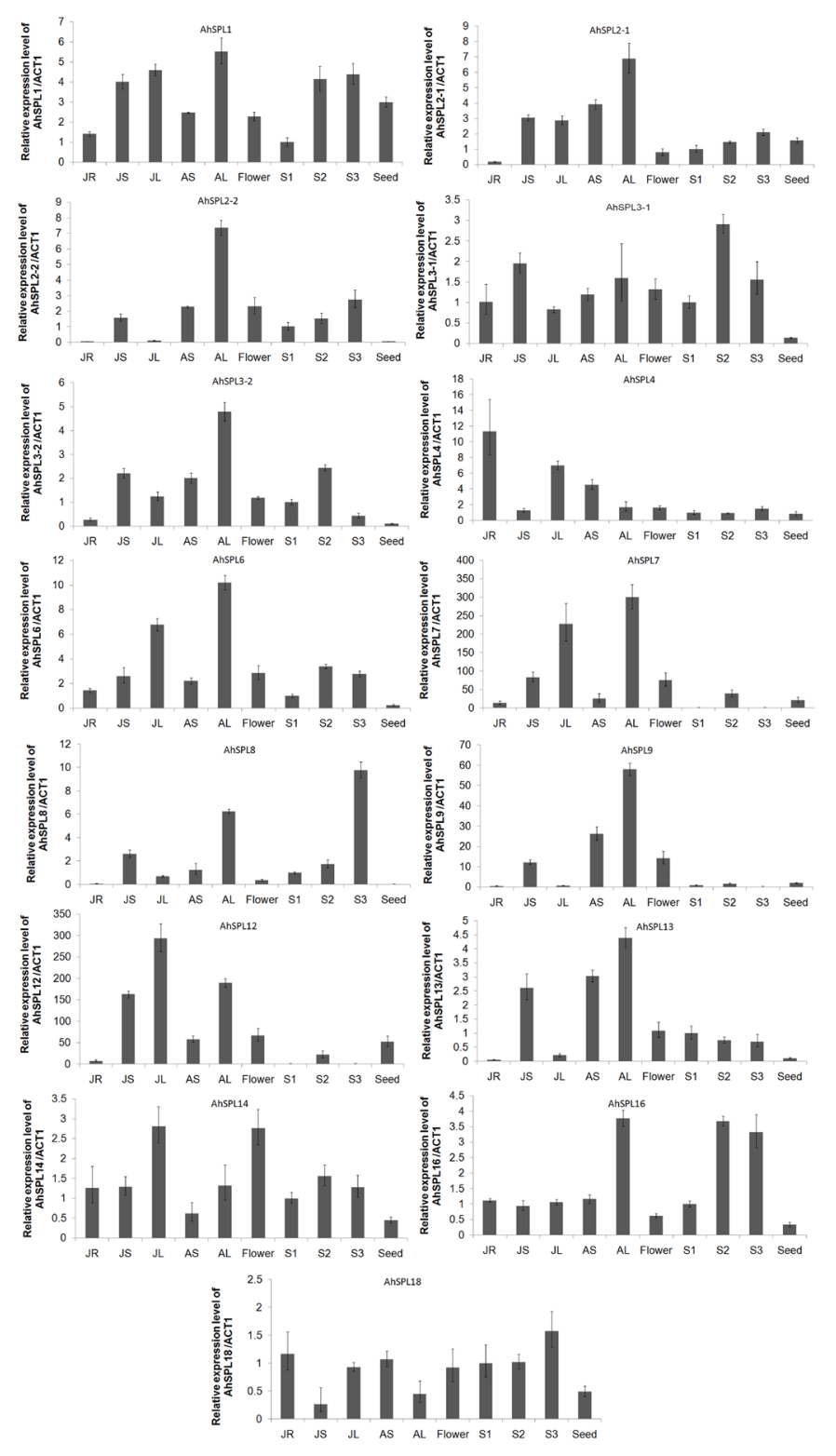

Figure 6. Temporal and spatial expression pattern of peanut SPL genes. JR: juvenile root; JS: juvenile stem; JL: juvenile leaf; AS: adult stem; AL: adult leaf; S1: aerial grown green gynophores; S2: gynophores buried in soil for about 3 days; S3: gynophores buried in the soil for about 9 days with enlarged pod; Seed: seed of mature stage. 
Previous studies have shown that the expression of some SPLs is differentially regulated during different developmental stages, such as the transition from the juvenile to the adult stage. Our results show that the expression levels of several peanut SPLs were significantly different between juvenile and adult leaves, e.g., AhSPL2-2 and AhSPL9 expression levels in adult leaves were over 70-fold higher than those in juvenile leaves. SPL2-1, SPL3-2, SPL8, SPL13, and SPL16 expression levels were over twice as high in adult leaves than in juvenile leaves. However, SPL4, SPL12, and SPL14 expression levels were about twice as high in juvenile leaves than in adult leaves (Figure 6). Significantly different expression levels between juvenile and adult stems were not found. Obvious morphological changes were observed during gynophore development from S1 to S3; most of the AhSPLs exhibited higher transcript levels in S2 than in S1. The AhSPL7 expression level at S2 was over 30-fold higher than that in S1, and SPL1, SPL3, SPL6, SPL7, SPL12, and SPL16 expression levels in S2 were 2-fold more than those in S1. From S2 to S3, the expression levels of some SPLs were maintained, while others decreased, e.g., SPL3, SPL7, SPL9, and SPL12. These results suggest that AhSPLs play critical roles during peanut pod development.

\section{DISCUSSION}

SBP-box genes encode a family of plant-specific transcription factors with no homologs in animals, humans, or bacteria (Cardon et al., 1999; Xie et al., 2006). SBP-box proteins can be divided into six (Hou et al., 2013) to eight (Li et al., 2013) groups. In our phylogenetic analysis, based on the alignment of the conserved DNA-binding domain, 107 SBP-box genes from six landplant species were clustered into six distinct groups (G1-G6). The thirteen members from the moss $P$. patens only clustered in G1, G4, and G5. The SBP-box gene family from other plant species was evolutionally more diverse. As expected, SPL proteins from peanut generally exhibited closer relationships to SBP-like proteins from dicotyledons than those from monocotyledons.

The expression levels of AhSPL1, AhSPL2-1, AhSPL2-2, AhSPL8, and AhSPL16 significantly increased after peanut gynophores penetrated the soil. However, the expression levels of these SPLs in mature seeds were very low. A similar phenomenon occurs in the grapevine; several SPLs are highly expressed during early fruit development, whereas during ripening their expression levels are much lower (Hou et al., 2013). Therefore, SPLs might play a crucial regulatory role during the early stages of peanut embryogenesis; further investigation is required to elucidate how these AhSPLs function during peanut embryo and pod development. In peanut, 7 of 15 SPLs contained sequences that were complementary to the mature miR156 sequence. Interestingly, all of the putative miR156-targeted AhSPLs were clustered in G2 and G3. The target sites of six SPLs were in the coding region downstream of the SBP domain, while the AhSPL2-1 target site was located in the 3' UTR. These results demonstrate that miR156/157 could downregulate the expression of these SPLs by mRNA degradation and translation inhibition, as reported in Arabidopsis (Wu and Poethig, 2006). However, miR156a, the only abundantly expressed member of the family in gynophores, exhibited similar expression levels in S1, S2, and S3 gynophores. The peanut miR157 expression level in S2 gynophores was three times higher than that in S1 gynophores (data not shown), suggesting that miRNA-regulated SPL expression may play a critical role during pod development. Further studies are required to elucidate the mechanism involved in miR156/157-targeted AhSPLs during peanut embryogenesis and pod development.

Peanut pod swelling is a complicated process that is controlled by several plant hormones, such as auxin, GA, ethylene, and abscisic acid (Xia et al., 2013). Previous studies have shown that AtSPL3 and AtSPL8 affect endogenous GA levels and signaling in Arabidopsis. Constitutive 
overexpression of SPL8 suppresses stem elongation and rosette leaf expansion, and delays flowering. These phenotypes could be rescued by the application of exogenous GA (Zhang et al., 2007), suggesting that SPL8 expression could reduce endogenous GA levels. A recent study demonstrated that DELLA proteins interact with SPLs to repress plant stem elongation and floral transition in Arabidopsis (Yu et al., 2012). We found that gynophore $\mathrm{GA}_{4 / 7}$ levels significantly decreased after gynophore soil penetration (data not shown). Zhu et al. (2014) reported that one to two days of dark treatment significantly decreases the endogenous $\mathrm{GA}_{3}$ content of gynophores. In the present study, the peanut SPL8 expression level was upregulated in dark-grown S2 (2-fold) and S3 (5-fold) gynophores in comparison with that in S1 gynophores (Figure 6). The decreased GA level in dark-grown gynophores could result in the accumulation of DELLA proteins and promote an interaction between DELLA and SPL proteins, which might reduce gynophore elongation and initiate pod development. It would be interesting to investigate how SPLs interact with GA biosynthesis and signaling in peanut embryonic development.

The constitutive expression of miR156-resistant SPL13 upregulates the expression of the ACC oxidase gene, suggesting the involvement of SPL13 in ethylene biosynthesis (Martin et al., 2010). However, ethylene treatment could also regulate SPL expression, e.g., the exogenous application of ethylene upregulated 12 apple SPLs and downregulated one apple SPL (Li et al., 2013). In the grapevine, ethylene treatment upregulated six SPLs and downregulated another six SPLs (Hou et al., 2013). In peanut, the ethylene content of S2 and S3 gynophores is several times higher than that in S1 gynophores (Shlamovitz et al., 1995). This increase in ethylene content in peanut is coincident with the upregulation of several SPLS. Are these genes directly regulated by ethylene, as in the apple and grapevine, or are they developmentally regulated? This is an interesting question, and further experiments are required to clarify the relationship between $S P L$ expression and ethylene accumulation in peanut gynophores.

$L F Y, F U L$, and $A P 1$ are meristem identity genes. SPL3, together with other miR156targeted $S P L$ transcription factors, directly activates these genes to control reproductive transition and meristem identity transition in Arabidopsis (Yamaguchi et al., 2009). Before soil penetration, peanut gynophores grow in a positively geotropic manner toward the ground, in a similar manner as roots. An intercalary meristem is located at the top of the gynophores. Soon after soil penetration, the growth of the gynophores ceases and pod enlargement is initiated, possibly due to changes in meristem activity. Whether this change in meristem activity is related to the upregulation of SPLs is an interesting possibility. Furthermore, a recent study demonstrated that SPL8 and other miR156targeted SPLs are key regulators of gynoecium development (Xing et al., 2013), suggesting that $S P L s$ may play important roles in peanut pod development.

\section{Conflicts of interest}

The authors declare no conflict of interest.

\section{ACKNOWLEDGMENTS}

Research supported by the Shandong Province Germplasm Innovation and Utilization Project, the National High Technology Research and Development Program of China (\#2013AA102602), the National Natural Science Foundation of China (\#31471526), Jinan Innovation Project (\#201102033), Shandong Provincial Natural Science Foundation (\#BS2013SW006), and the Young Talents Training Program of Shandong Academy of Agricultural Sciences. 


\section{REFERENCES}

Birkenbihl RP, Jach G, Saedler H and Huijser P (2005). Functional dissection of the plant-specific SBP-domain: overlap of the DNA-binding and nuclear localization domains. J. Mol. Biol. 352: 585-596. http://dx.doi.org/10.1016/j.jmb.2005.07.013

Cardon G, Höhmann S, Klein J, Nettesheim K, et al. (1999). Molecular characterisation of the Arabidopsis SBP-box genes. Gene 237: 91-104. http://dx.doi.org/10.1016/S0378-1119(99)00308-X

Chuck G, Whipple C, Jackson D and Hake S (2010). The maize SBP-box transcription factor encoded by tasselsheath4 regulates bract development and the establishment of meristem boundaries. Development 137: 1243-1250. http://dx.doi. org/10.1242/dev.048348

Gandikota M, Birkenbihl RP, Höhmann S, Cardon GH, et al. (2007). The miRNA156/157 recognition element in the 3' UTR of the Arabidopsis SBP box gene SPL3 prevents early flowering by translational inhibition in seedlings. Plant J. 49: 683-693. http://dx.doi.org/10.1111/j.1365-313X.2006.02983.x

Guo AY, Zhu QH, Gu X, Ge S, et al. (2008). Genome-wide identification and evolutionary analysis of the plant specific SBP-box transcription factor family. Gene 418: 1-8. http://dx.doi.org/10.1016/j.gene.2008.03.016

Hou H, Li J, Gao M, Singer SD, et al. (2013). Genomic organization, phylogenetic comparison and differential expression of the SBP-box family genes in grape. PLoS One 8: e59358. http://dx.doi.org/10.1371/journal.pone.0059358

Klein J, Saedler H and Huijser P (1996). A new family of DNA binding proteins includes putative transcriptional regulators of the Antirrhinum majus floral meristem identity gene SQUAMOSA. Mol. Gen. Genet. 250: 7-16.

Kropat J, Tottey S, Birkenbihl RP, Depège N, et al. (2005). A regulator of nutritional copper signaling in Chlamydomonas is an SBP domain protein that recognizes the GTAC core of copper response element. Proc. Natl. Acad. Sci. USA 102: 1873018735. http://dx.doi.org/10.1073/pnas.0507693102

Lännenpää M, Jänönen I, Hölttä-Vuori M, Gardemeister M, et al. (2004). A new SBP-box gene BpSPL1 in silver birch (Betula pendula). Physiol. Plant. 120: 491-500. http://dx.doi.org/10.1111/j.0031-9317.2004.00254.x

Li J, Hou H, Li X, Xiang J, et al. (2013). Genome-wide identification and analysis of the SBP-box family genes in apple (Malus × domestica Borkh.). Plant Physiol. Biochem. 70: 100-114. http://dx.doi.org/10.1016/j.plaphy.2013.05.021

Martin RC, Asahina M, Liu PP, Kristof JR, et al. (2010). The microRNA156 and microRNA172 gene regulation cascades at post-germinative stages in Arabidopsis. Seed Sci. Res. 20: 79-87. http://dx.doi.org/10.1017/S0960258510000085

Miura K, Ikeda M, Matsubara A, Song XJ, et al. (2010). OsSPL14 promotes panicle branching and higher grain productivity in rice. Nat. Genet. 42: 545-549. http://dx.doi.org/10.1038/ng.592

Moreno MA, Harper LC, Krueger RW, Dellaporta SL, et al. (1997). liguleless1 encodes a nuclear-localized protein required for induction of ligules and auricles during maize leaf organogenesis. Genes Dev. 11: 616-628. http://dx.doi.org/10.1101/ gad.11.5.616

Rhoades MW, Reinhart BJ, Lim LP, Burge CB, et al. (2002). Prediction of plant microRNA targets. Cell 110: 513-520. http:// dx.doi.org/10.1016/S0092-8674(02)00863-2

Riese M, Höhmann S, Saedler H, Münster T, et al. (2007). Comparative analysis of the SBP-box gene families in $P$. patens and seed plants. Gene 401: 28-37. http://dx.doi.org/10.1016/i.gene.2007.06.018

Salinas M, Xing S, Höhmann S, Berndtgen R, et al. (2012). Genomic organization, phylogenetic comparison and differential expression of the SBP-box family of transcription factors in tomato. Planta 235: 1171-1184. http://dx.doi.org/10.1007/ s00425-011-1565-y

Schwarz S, Grande AV, Bujdoso N, Saedler H, et al. (2008). The microRNA regulated SBP-box genes SPL9 and SPL15 control shoot maturation in Arabidopsis. Plant Mol. Biol. 67: 183-195. http://dx.doi.org/10.1007/s11103-008-9310-z

Shlamovitz N, Ziv M and Zamski E (1995). Light, dark and growth regulator involvement in groundnut (Arachis hypogaea L.) pod development. Plant Growth Regul. 16: 37-42. http://dx.doi.org/10.1007/BF00040505

Shikata M, Koyama T, Mitsuda N and Ohme-Takagi M (2009). Arabidopsis SBP-box genes SPL10, SPL11 and SPL2 control morphological change in association with shoot maturation in the reproductive phase. Plant Cell Physiol. 50: 2133-2145. http://dx.doi.org/10.1093/pcp/pcp148

Tamura K, Dudley J, Nei M and Kumar S (2007). MEGA4: Molecular evolutionary genetics analysis (MEGA) software version 4.0. Mol. Biol. Evol. 24: 1596-1599. http://dx.doi.org/10.1093/molbev/msm092

Thompson JD, Higgins DG and Gibson TJ (1994). CLUSTAL W: improving the sensitivity of progressive multiple sequence alignment through sequence weighting, position-specific gap penalties and weight matrix choice. Nucleic Acids Res. 22: 4673-4680. http://dx.doi.org/10.1093/nar/22.22.4673

Thompson LK, Ziv M and Deitzer GF (1985). Photocontrol of peanut (Arachis hypogaea L.) embryo and ovule development in vitro. Plant Physiol. 78: 370-373. http://dx.doi.org/10.1104/pp.78.2.370

Unte US, Sorensen AM, Pesaresi P, Gandikota M, et al. (2003). SPL8, an SBP-box gene that affects pollen sac development in Arabidopsis. Plant Cell 15: 1009-1019. http://dx.doi.org/10.1105/tpc.010678 
Usami T, Horiguchi G, Yano S and Tsukaya H (2009). The more and smaller cells mutants of Arabidopsis thaliana identify novel roles for SQUAMOSA PROMOTER BINDING PROTEIN-LIKE genes in the control of heteroblasty. Development 136: 955-964. http://dx.doi.org/10.1242/dev.028613

Voinnet O (2009). Origin, biogenesis, and activity of plant microRNAs. Cell 136: 669-687. http://dx.doi.org/10.1016/j. cell.2009.01.046

Wang JW, Park MY, Wang LJ, Koo Y, et al. (2011). miRNA control of vegetative phase change in trees. PLoS Genet. 7: e1002012. http://dx.doi.org/10.1371/journal.pgen.1002012

Wang S, Wu K, Yuan Q, Liu X, et al. (2012). Control of grain size, shape and quality by OsSPL16 in rice. Nat. Genet. 44: 950954. http://dx.doi.org/10.1038/ng.2327

Weigel D, Alvarez J, Smyth DR, Yanofsky MF, et al. (1992). LEAFY controls floral meristem identity in Arabidopsis. Cell 69: 843-859. http://dx.doi.org/10.1016/0092-8674(92)90295-N

Wu G and Poethig RS (2006). Temporal regulation of shoot development in Arabidopsis thaliana by miR156 and its target SPL3. Development 133: 3539-3547. http://dx.doi.org/10.1242/dev.02521

Wu G, Park MY, Conway SR, Wang JW, et al. (2009). The sequential action of miR156 and miR172 regulates developmental timing in Arabidopsis. Cell 138: 750-759. http://dx.doi.org/10.1016/j.cell.2009.06.031

Xia H, Zhao C, Hou L, Li A, et al. (2013). Transcriptome profiling of peanut gynophores revealed global reprogramming of gene expression during early pod development in darkness. BMC Genomics 14: 517. http://dx.doi.org/10.1186/1471-2164-14-517

Xie K, Wu C and Xiong L (2006). Genomic organization, differential expression, and interaction of SQUAMOSA promoterbinding-like transcription factors and microRNA156 in rice. Plant Physiol. 142: 280-293. http://dx.doi.org/10.1104/ pp. 106.084475

Xing S, Salinas M, Höhmann S, Berndtgen R, et al. (2010). miR156-targeted and nontargeted SBP-box transcription factors act in concert to secure male fertility in Arabidopsis. Plant Cell 22: 3935-3950. http://dx.doi.org/10.1105/tpc.110.079343

Xing S, Salinas M, Garcia-Molina A, Höhmann S, et al. (2013). SPL8 and miR156-targeted SPL genes redundantly regulate Arabidopsis gynoecium differential patterning. Plant J. 75: 566-577. http://dx.doi.org/10.1111/tpj.12221

Yamaguchi A, Wu MF, Yang L, Wu G, et al. (2009). The microRNA-regulated SBP-Box transcription factor SPL3 is a direct upstream activator of LEAFY, FRUITFULL, and APETALA1. Dev. Cell 17: 268-278. http://dx.doi.org/10.1016/j. devcel.2009.06.007

Yamasaki H, Hayashi M, Fukazawa M, Kobayashi Y, et al. (2009). SQUAMOSA promoter-binding protein-like7 is a central regulator for copper homeostasis in Arabidopsis. Plant Cell 21: 347-361. http://dx.doi.org/10.1105/tpc.108.060137

Yu S, Galvão VC, Zhang YC, Horrer D, et al. (2012). Gibberellin regulates the Arabidopsis floral transition through miR156targeted SQUAMOSA promoter binding-like transcription factors. Plant Cell 24: 3320-3332. http://dx.doi.org/10.1105/ tpc.112.101014 PubMed

Zhang Y, Schwarz S, Saedler H and Huijser P (2007). SPL8, a local regulator in a subset of gibberellin-mediated developmental processes in Arabidopsis. Plant Mol. Biol. 63: 429-439. http://dx.doi.org/10.1007/s11103-006-9099-6

Zhu W, Chen X, Li H, Zhu F, et al. (2014). Comparative transcriptome analysis of aerial and subterranean pods development provides insights into seed abortion in peanut. Plant Mol. Biol. 85: 395-409. http://dx.doi.org/10.1007/s11103-014-0193-X

\section{Supplementary material}

Table S1. SBP-domain sequences and accession numbers of selected SBP-box genes used for phylogenetic analysis.

Table S2. Quantitative reverse transcription-polymerase chain reaction primer sequences.

Table S3. Consensus sequences of the SBP motifs in Arachis hypogaea proteins ${ }^{\text {. }}$.

www.geneticsmr.com/year2016/vol2016/vol15-1/pdf/gmr7344_supplementary.pdf 